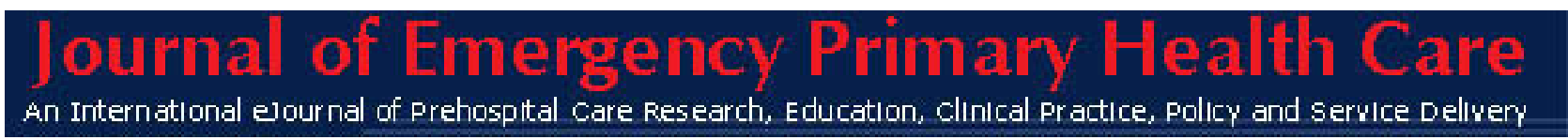

1447-4999

\title{
EDITORIAL
}

\section{DEVELOPING AN INTERNATIONAL CONSENSUS ON RESUSCITATION SCIENCE}

Article No. 990065

Ian Jacobs

The publication of "International Guidelines 2000 for CPR and ECC - A Consensus on Science”(1) has been widely acknowledged amongst those involved in the teaching, practice and research of resuscitation. It is extensively used by many organisations, including the Australian Resuscitation Council (ARC), as a basis on which resuscitation guidelines may be developed.

The development of Guidelines 2000 represented a significant milestone in this area, in that 1) it endeavoured to apply a systematic evidence based approach in quantifying the science of resuscitation and, 2) there was significant international involvement. It was the first time an international conference had been held to develop guidelines for resuscitation. A full description of the guidelines 2000 process has been published elsewhere and I would recommend readers review this document.(2) In addition, a discussion of the evidence that was evaluated for specific resuscitation topics has also been published in the conference proceedings.(3)

While the Guidelines 2000 Conference achieved much in achieving consensus on international resuscitation guidelines the process was not without significant challenges. Much was learnt as to how the process may be further enhanced and refined for future guideline iterations. Of significance in the review process was the realisation that whilst there is "common resuscitation science" this may not be the case for guidelines, as the latter often requires issues of feasibility and implementation to be considered.

Under the auspices of the International Liaison Committee on Resuscitation (ILCOR), an update of Guidelines 2000 is well underway. The process - Consensus 2005 -involves considerable international involvement and addresses many of the issues raised during the Guidelines 2000 process. The published document will focus primarily on the science of resuscitation and be much shorter, approximately 300 pages less than Guidelines 2000. While some treatment recommendations will be made these will not serve as complete guidelines. It is anticipated that each National or Regional Resuscitation Council will use the common science outlined in the Consensus 2005 document on which to develop resuscitation guidelines appropriate to their region.

To accomplish the task ahead, ILCOR meets twice yearly and oversees the entire process. In addition, there are a number of Task Forces in the following areas.

- Basic Life Support

- Advanced Life Support

- Paediatric Life Support

- Neonatal Life Support

- Interdisciplinary

Each Task Force has two chairs (one USA and one international) who are responsible for managing the evidence-based review process within their respective groups including finding topic reviewers.

Author(s) Ian Jacobs 


\section{Journal of Emergency Primary Health Care (JEPHC), Vol.1, Issue3-4, 2003}

There are over 250 topics currently being reviewed with each topic being assigned to at least two reviewers (one international and one from the USA). Each reviewer independently undertakes a comprehensive literature search and appraises the evidence pertaining to that topic. At a minimum, each reviewer would have searched the Cochrane Database of Systematic Reviews, the Cochrane Central Register of Controlled Trials, MEDLINE and EMBASE. An evidence based template or worksheet has been developed which assists the reviewer to assess both the quality and level of the evidence reviewed. This part of the evidence evaluation process concludes with each of the two reviewers coming to consensus on the evidence reviewed for that specific topic.

To monitor the quality of the evidence review process, two evidence evaluation co-ordinators have been appointed. Consistent with the entire evidence evaluation process there is one international and one USA based co-ordinator. Their responsibility is to ensure a high and consistent standard of evidence-based reviews on which the final Consensus on Science 2005 document will be based.

Each of the topics reviewed will be presented at the Consensus 2005 Conference to be held in Dallas in January 2005. This conference will provide an opportunity for the evidence to be peer reviewed by experts from the international resuscitation community prior to its publication. Again it should be noted that the process is one of science evaluation with resuscitation guidelines being developed by various national resuscitation councils.

Development of the final Consensus of Resuscitation Science document will be completed during the first half of 2005 with publication soon thereafter. For each of the treatment recommendations, a level of evidence for that recommendation will also be published. Furthermore the evidence evaluation worksheets will also be available via the web.

In addition to the Task Forces outlined above, a further three have been established in the areas of:

- Acute Myocardial Infarction / Acute Coronary Syndromes

- Stroke

- First Aid

These are undertaken under the auspices of the American Heart Association (AHA) with varying degrees of involvement from the international resuscitation community or ILCOR. Wherever possible the ARC is actively engaged in reviews in these topic areas.

In summary, the evidence evaluation of the science of resuscitation is an ongoing, dynamic and a work in progress. Under the guidance of ILCOR, to which the ARC is a major contributor, international consensus on science in this area can be developed. The final document will have minimal guideline content and focus on the science common to all. It will be the responsibility of organisations such as the ARC to develop resuscitation guidelines which are appropriate and sensitive to local needs.

\section{References:}

1. American Heart Association in collaboration with International Liaison Committee on Resuscitation. Guidelines for Cardiopulmonary Resuscitation and Emergency Cardiovascular Care--An International Consensus on Science. Resuscitation 2000;46(1-3):3-430.

2. Part 1: introduction to the International Guidelines 2000 for CPR and ECC. A consensus on science. Resuscitation 2000;46(1-3):3-15.

3. Proceedings of the Guidelines 2000 Conference for Cardiopulmonary Resuscitation and Emergency Cardiovascular Care: An International Consensus on Science. Ann Emerg Med 2001;37(4 Suppl):S1-S200.

Author(s) Ian Jacobs 